\title{
DISPERSIVE ORDERING OF DISTRIBUTIONS
}

\author{
D. J. SAUNDERS, ${ }^{*}$ British Broadcasting Corporation
}

1. Shaked (1982) gives a characterisation of dispersive ordering for distributions which are absolutely continuous with interval supports. However there is a technical error in his proof of the second part of Theorem 2.1, although the result is still true. The difficulty arises because zero terms are discarded in the definition of the sign change operator $S$. Therefore the equality of $F_{c_{\alpha}}^{\cdot}\left(x^{\prime}\right)$ and $G\left(x^{\prime}\right)$ does not imply that $F_{c_{\alpha}}(x) \geqq$ $G(x) \forall x>x^{\prime}$.

A suitable amendment to the proof is to choose $\varepsilon>0$ and define

Then

$$
c_{\alpha, \varepsilon}=G^{-1}(\alpha)-F^{-1}(\alpha)-\varepsilon .
$$

$$
F_{c_{\alpha, \varepsilon}}\left(x^{\prime}\right)=F\left(G^{-1}(\alpha)-c_{\alpha, \varepsilon}\right)=F\left(F^{-1}(\alpha)+\varepsilon\right)>\alpha=G\left(x^{\prime}\right)
$$

since $F$ is strictly increasing at $F^{-1}(\alpha)$. Hence (using 2.2)

$$
F_{c_{\alpha, \varepsilon}}(x) \geqq G(x) \quad \forall x>x^{\prime} .
$$

The argument of the original proof then shows that, $\forall \beta>\alpha$,

$$
G^{-1}(\beta)-F^{-1}(\beta) \geqq c_{\alpha, \varepsilon}=G^{-1}(\alpha)-F^{-1}(\alpha)-\varepsilon .
$$

Since this holds for every $\varepsilon>0$, the result follows.

2. The relationship of ordering in dispersion can be described, loosely, by saying that the graph of one distribution is always 'steeper' than the graph of the other at points with corresponding image values, where the definition of 'steeper' is given in terms of conditions on the inverse functions. The Shaked characterisation then says that one distribution is always 'steeper' than the other if, and only if, you can never make the two graphs cross in the wrong direction by sliding one graph horizontally. This is then a straightforward property of certain real-valued functions, and one would expect the proof to use elementary properties of the real numbers. The proof given by Shaked, for absolutely continuous distribution functions with interval supports, is of this kind.

Lynch, Mimmack and Proschan (1983) extend the result to general distribution functions. However, in one direction their proof uses approximations to a general distribution by continuous distributions, and requires several rather deeper results. An elementary proof would therefore seem desirable, and one is given below.

Theorem. If $\forall c \in R$, (a) $S\left(F_{c}-G\right) \leqq 1$ and (b) if $S\left(F_{c}-G\right)=1$ then the sign sequence is,-+ then $F \underset{\text { disp }}{\leqq}$.

Proof. First, (a) implies (either $F \underset{\text { disp }}{\leqq} G$ or $G \underset{\text { disp }}{\leqq} F$ ). For if not, then there are $\alpha_{i}, \beta_{i} \in$

Received 19 March 1984; revision received 13 June 1984.

* Postal address: British Broadcasting Corporation, Open University Production Centre, Walton Hall, Milton Keynes, MK7 6BH, UK. 
$(0,1)$ with $\alpha_{i}<\beta_{i}(i=1,2)$ and

$$
\begin{aligned}
& F^{-1}\left(\beta_{1}\right)-F^{-1}\left(\alpha_{1}\right)>G^{-1}\left(\beta_{1}\right)-G^{-1}\left(\alpha_{1}\right) \\
& F^{-1}\left(\beta_{2}\right)-F^{-1}\left(\alpha_{2}\right)<G^{-1}\left(\beta_{2}\right)-G^{-1}\left(\alpha_{2}\right) .
\end{aligned}
$$

Let $\gamma_{1}, \gamma_{2}, \gamma_{3}, \gamma_{4}$ be $\alpha_{1}, \beta_{1}, \alpha_{2}, \beta_{2}$ arranged in ascending order. Note that at least three of these values must be distinct, and that the sequence $F^{-1}\left(\gamma_{i}\right)-G^{-1}\left(\gamma_{i}\right), i=1,2,3,4$ is neither always increasing nor always decreasing. So suppose without loss of generality

$$
F^{-1}\left(\gamma_{1}\right)-G^{-1}\left(\gamma_{1}\right)>F^{-1}\left(\gamma_{2}\right)-G^{-1}\left(\gamma_{2}\right)<F^{-1}\left(\gamma_{3}\right)-G^{-1}\left(\gamma_{3}\right)
$$

and write

$$
c=-\frac{1}{2}\left[\min \left\{F^{-1}\left(\gamma_{1}\right)-G^{-1}\left(\gamma_{1}\right), F^{-1}\left(\gamma_{3}\right)-G^{-1}\left(\gamma_{3}\right)\right\}+F^{-1}\left(\gamma_{2}\right)-G^{-1}\left(\gamma_{2}\right)\right] .
$$

Then

$$
F_{c}^{-1}\left(\gamma_{1}\right)>G^{-1}\left(\gamma_{1}\right), \quad F_{c}^{-1}\left(\gamma_{2}\right)<G^{-1}\left(\gamma_{2}\right), \quad F_{c}^{-1}\left(\gamma_{3}\right)>G^{-1}\left(\gamma_{3}\right) .
$$

Write $x_{1}=G^{-1}\left(\gamma_{1}\right), x_{2}=F_{c}^{-1}\left(\gamma_{2}\right), x_{3}=G^{-1}\left(\gamma_{3}\right)$. Then $x_{1}<x_{2}<x_{3}$, but

$$
F_{c}\left(x_{1}\right)<G\left(x_{1}\right), \quad F_{c}\left(x_{2}\right)>G\left(x_{2}\right), \quad F_{c}\left(x_{3}\right)<G\left(x_{3}\right) .
$$

Consequently $S\left(F_{\mathrm{c}}-G\right) \geqq 2$.

Since it is easy to see that $S\left(F_{c}-G\right)=0 \forall c \in R$ implies that $\exists c \in R$ with $F_{c}=G$ (so

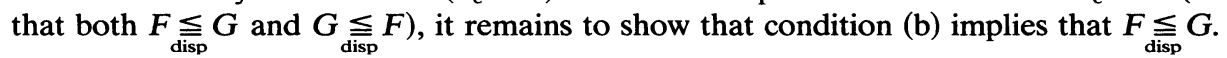

So suppose that, for some $c \in R, S\left(F_{c}-G\right)=1$, with the sign change from - to + . Then there are $x, y \in R, x<y$ with

$$
F_{\mathrm{c}}(x)<G(x), \quad F_{\mathrm{c}}(y)>G(y) .
$$

Let $\alpha=G(x), \beta=F_{c}(y)$. Then

$$
G^{-1}(\beta)-G^{-1}(\alpha)>F_{c}^{-1}(\beta)-F_{c}^{-1}(\alpha)=F^{-1}(\beta)-F^{-1}(\alpha)
$$

showing that $G \underset{\text { disp }}{\leqq} F$.

\section{References}

Lynch, J., Mimmack, G. And Proschan, F. (1983) Dispersive ordering results. Adv. Appl. Prob. 15, 889-891.

Shaked, M. (1982) Dispersive ordering of distributions. J. Appl. Prob. 19, 310-320. 